\title{
An Audible Search Engine for Visually Impaired Users: A Prototype Developed using Assistive Technology
}

\author{
Arfa Masood \\ Department of Computer \\ Science \\ LCWU \\ Lahore, Pakistan \\ Mubashra Alam \\ Department of Computer \\ Science \\ LCWU \\ Lahore, Pakistan
}

\author{
Mariam Rehman \\ Department of Computer \\ Science \\ LCWU \\ Lahore, Pakistan
Sonia Kanwal
Department of Computer
Science
LCWU
Lahore, Pakistan

\author{
Maria Anjum \\ Department of Computer \\ Science \\ LCWU \\ Lahore, Pakistan
Muhammad Rafiq Mufti
Department of Computer
Science
COMSATS
Vehari, Pakistan

\begin{abstract}
The objective of the research work was to create an Audible Search Engine for Visually Impaired Users, in order to improve their accessibility to find their desired results. In order to fulfill the desired objective, the preliminary study was performed, based on the observation conducted with the blinds that used assistive technology to navigate the Web. For better Speech Recognition, Web Crawler and Speech Synthesizer technologies have been adopted. The designed system detects user's speech and then converts speech into text. Moreover, Web Crawler makes relevant links and titles available in a short span. Hence, the Speech Synthesizer read, enable the results converted into an audio format which are understandable by visually impaired users. The research work focuses on easier, efficient and less frustrating access for sightless individuals.
\end{abstract}

\section{Keywords}

Visually Impaired; Assistive technology; Speech Recognition; Web Crawler; Speech Synthesizer, Audible Search Engine

\section{INTRODUCTION}

For effective interaction, search engines are the programs that become fundamental tool for retrieval of information on the World Wide Web. However, visually impaired users find difficulties while exploring the web pages on internet and it requires more time. In order to facilitate visually impaired users, the screen readers are used in order to reduce their searching time. To facilitate visually impaired users in searching information on internet by developing search engine facility, a desktop application has been introduced which provides speech recognizer and speech synthesizer technology by using audio facility [1]. The main objectives of this research work are:

- To review the existing literature in order to understand the components of speech recognition and speech synthesizer to provide assistance for visually impaired users.

- To develop software application which is mainly used for speech recognition, speech synthesizer, text generation and conversion in audio, web crawling and tool provides different audio features i.e. Start, Pause, Replay and Stop.

- To enhance the usability of software application by focusing on simplicity, rapid access, navigation links and layout of search results.

\section{LITERATURE REVIEW}

In last few decades, in order to index large amount of heterogeneous data accessible on internet, search technology has been evolved from collection of homogeneous data. Almost 161 million persons live with a disabling visual impairment among which 37 million are blind. In developing countries of Africa, Asia, Latin America and the Pacific Regions, $90 \%$ of these people live there [2].

Manzeet and Parteek's worked on conversion of English and Hindi text to Braille. To teach blind people in their research, they discussed all the possible ways to teach Braille through audio aids [3]. They focused on different approaches and problems related to particular patterns which blinds were using to learn Braille [2].

Reena and Geetanjali made an attempt to develop a Speech Recognition and Synthesis Tool (SRST) as assistive technology and provide a solution for communication between physically disabled persons; majorly focused on blind and deaf [4]. According to their observation, both blind and deaf users can communicate to each other efficiently only in noise free environment. To become proficient with tool commands and speech recognition commands, blind students take more time than deaf students. Nurulisma and Halimah highlighted virtual learning environment to facilitate the visually impaired and blind learners. They used voice recognition browser as a tool [5].

For designing search engine user interfaces, Patrizia et al. formulate eight guidelines that are based on a preliminary study on usability and accessibility of search tools. While maintaining the same 'look \& feel', they modified the source code of Google's interface. They demonstrated that with very little effort, it is possible to make access more efficient, easier and less frustrating for sightless individuals [6].

For improving the design of search engine interfaces, Barbara et al. proposed specific and appropriate guidelines. They also discussed the most significant differences between an aural perception and visual layout. Furthermore, by means of a screen reader or voice synthesizer, they describe the main design issues affecting the user interface of a search engine when a sightless user interacts [7]. They also discussed accessibility and usability requirements of interface for 
sightless persons. In their work, they concluded that both aspects are of great importance for navigation via assistive devices.

Loo et al. make an effort to work on the design of a new human-computer interface model and an associated computer system for visually impaired people so that they can be able to browse the World Wide Web using Internet [1]. For blind computer users, Winberg discussed three stages of interaction. The first stage is concerned with the investigation performed on access of audio interface. The second stage is linked to collaboration between sighted and blind computer users. However, the final stage is concerned about usage of assistive technology for blind users [8]. Voice based Search Engine and Web-page Reader was introduced by Ummuhanysifa et al. which allows the users to command and control the web browser through their voice [9].

Al-Rehili et al. focused on the design, testing, benefits and analysis of a desktop application. This application is able to translate English text to Arabic text, pronounce english and / or arabic text. It is also capable of recognizing the english speech to convert it into a corresponding English text. It is helpful for users to complete their tasks easily especially those with special needs [10]. Karthik et al. described voice enabled ontology based search engine for blinds. They used a search engine that is conceptual and receives the user query via voice and converts them into text with the help of software [11]. An interesting research focusing on search engine design for blinds was done by Ferworn and his co-members. To organize results into a voice menu format, this research discusses the implementation of an auditory search engine prototype. It provides vocal output by using real-time textcategorization [12].

Yang et al. worked on specialized design of web search for the blind people. For comparison, Google search engine was taken as a base search engine. Specialized Search Engine for the Blind (SSEB) was designed with an improved functions accessible interface and (user-centered functions, searching assistance functions, and specialized design for the blind). To verify the effect of SSEB, an experiment was conducted. With the experimental results, it was found that users attained better performance in SSEB than that of Google. Moreover, users showed higher satisfaction with SSEB also. Hence, it is proved from this research that consideration for designing an accessible website for the blind users is very significant. It is also expected that the users group of SSEB can be extended to all visual-impaired people. Thus, all people can keep pace with the changing World Wide Web, and can be able to use all internet resources without limitations and disabilities [13][14].

Singh et al. performed a detailed study on recognition and hidden smarkov model. To develop a voice based user machine interface system, this model was carried out. Basically, this application can be linked to disable persons who are unable to operate computer through mouse and keyboard. Such kind of persons can use computer with Automatic Speech Recognition system. In addition, users can operate system with their own speech by using this system. The second application is linked to those users who are not comfortable with English languages and feel easy to work with their native languages i.e. Hindi, English, and Punjabi [15].

\section{METHODOLOGY}

The current study collected experimental data from visually impaired users. In addition, subjective opinions were also gathered in order to make improvement in the design of search engine. Furthermore, formal system design procedure was applied in order to develop the system. The Google Search API and Speech API's were selected for constructing the audible search engine. The in-depth interviews were conducted in order to highlight the needs of visually impaired users. Later, architecture of audible search engine was designed for visually impaired users which contain all the features they need for searching results on Web. Moreover, development was started, an Audible Search Engine for Visually Impaired Users by using speech recognition and speech synthesizer technology. Finally, evaluation was performed with visually impaired users of independent center at LCWU. The methodological flow is shown in Fig. 1 which consists of five steps. These steps have been explained below in detail.

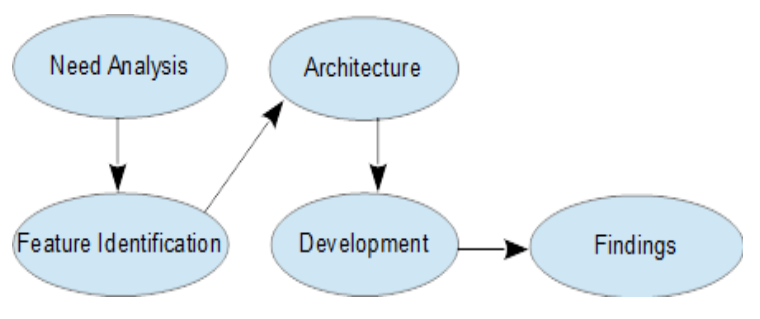

Figure 1. System Methodology

\subsection{Need Analysis}

The problem encountered by visually impaired users while using the internet can be classified into four groups. The operational need is shown as the solution to each problem. Two blind persons who were undergraduate students, frequently used both the internet and search engines, took part in the interviews. They argued that search results for blind people should not contain complicated structures and repeated functional items. The most important element should be put in the forefront of a search result to prevent users from wasting their time on non informative items. The searched links should avoid complicated structure, such as link details, HTML tags, images, videos or other symmetrical areas. Most essential for a Web link for blind users is to provide sufficient information with clear navigation concepts through audio aid.

\subsection{Identification of Features}

According to different methods of system design and development, operational need (see TABLE I.) and system requirement (see TABLE II.) were considered to develop a system for visually impaired users. Then, main functions were designed for visually impaired users.

Table 1. Visually Impaired Operational Needs

\begin{tabular}{|c|c|c|}
\hline & Problem & Solution \\
\hline Hardware & Usage of mouse & $\begin{array}{l}\text { Short cut keys are } \\
\text { being used instead } \\
\text { of mouse }\end{array}$ \\
\hline Software & 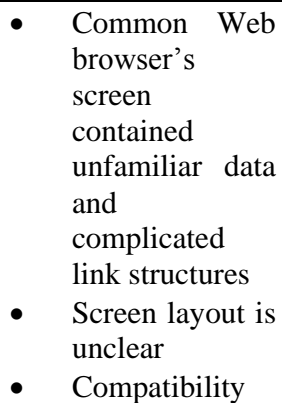 & 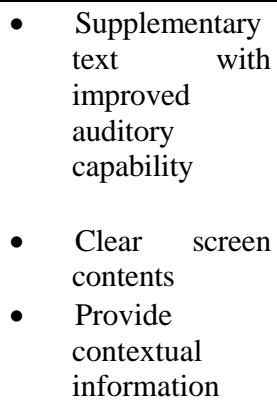 \\
\hline
\end{tabular}




\begin{tabular}{|c|c|c|}
\hline & $\begin{array}{l}\text { issues between } \\
\text { screen reader } \\
\text { and web links }\end{array}$ & $\begin{array}{l}\text { without the use } \\
\text { of screen } \\
\text { reader } \\
\text { Ensure that } \\
\text { Search results } \\
\text { media } \\
\text { accessibility } \\
\text { featuring } \\
\text { auditory format } \\
\text { gracefully }\end{array}$ \\
\hline $\begin{array}{c}\text { Navigation } \\
\text { Concepts }\end{array}$ & $\begin{array}{l}\text { Confusing } \\
\text { navigation, such as, } \\
\text { scrolling of links etc. }\end{array}$ & $\begin{array}{l}\text { Clear Identification } \\
\text { of target links }\end{array}$ \\
\hline
\end{tabular}

Table 2. System Requirement for Visually Impaired Users

\begin{tabular}{|l|l|}
\hline \multicolumn{1}{|c|}{$\begin{array}{c}\text { Visually Impaired User } \\
\text { Characteristics }\end{array}$} & \multicolumn{1}{|c|}{ System Requirements } \\
\hline No physical disabilities & Use keyboard to input data \\
\hline No Hearing disabilities & I/O data through audio media \\
\hline $\begin{array}{l}\text { Blind and no hearing } \\
\text { disabilities }\end{array}$ & $\begin{array}{l}\text { Input data through audio and } \\
\text { for navigation, keyboard is } \\
\text { used }\end{array}$ \\
\hline $\begin{array}{l}\text { No prior knowledge of } \\
\text { computer usage }\end{array}$ & $\begin{array}{l}\text { Provide training and guidance } \\
\text { in order to execute software } \\
\text { application }\end{array}$ \\
\hline Without keyboard experience & $\begin{array}{l}\text { Provide instructions to the } \\
\text { users }\end{array}$ \\
\hline
\end{tabular}

\subsection{Architecture}

Architectural overview of an Audible Search Engine for Visually Impaired User is being described at two levels i.e. non-technical architecture and technical architecture for Audible Search Engine for Visually Impaired Users.

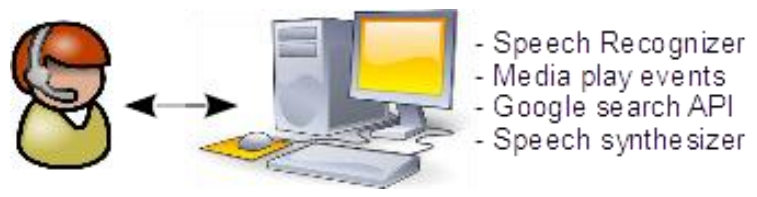

Figure 2. Search Engine Facilities

\subsection{Product Function}

The system can function in two modes namely speech recognition and speech synthesizer mode. The system will be in speech recognition mode, when user will input in the form of audio and speech recognition module will convert audio input into text. The second mode is speech synthesizer which converts audio input into text format. Moreover, speech synthesizer mode is accepting search results from web crawler and converts the listed data into text format. Finally, speech synthesizer converts this input into audio format in order to make it understandable for visually impaired users

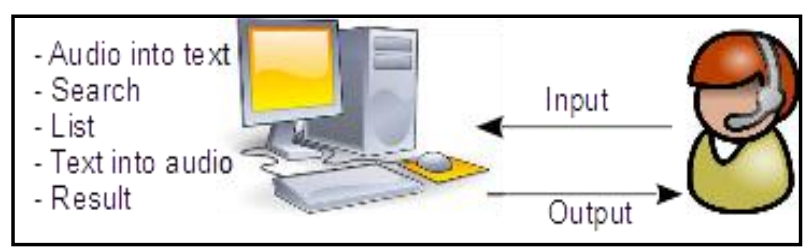

Figure 3. Search Engine Processes

\subsection{System Components}

\subsubsection{Speech Recognition Mode}

The main actor of the system is visually impaired users. In order to facilitate them, the user audio input is taken by microphone. The module of speech recognition recognizes that input and load audio to text by speech recognition engine. The text input is matched by the database dictionary. If it is matched, then the matched words are sent to Google API.

\subsubsection{Web Crawler}

The Google API uses web crawler to search the text query input into the database. The query will be in continues vocabulary. If the searched data matched by the input query, then web crawler creates an index of URL's. If URL's matched to already created index or does not exist in index, the search results will be displayed in a listed format. User will select the listed search results according to his / her requirement. Finally, the system will read these search results in audio format.

public static IList $<$ Result $>$ Search(string query) \{

Console.WriteLine("Executing google custom search for query: $\{0\} \ldots$. , query); CseResource.ListRequest listRequest $=$ Service.Cse.List(query); listRequest.Cx = cx; Search search = listRequest.Execute(); return search.Items;

\subsubsection{Speech Synthesizer Mode}

The speech synthesizer converts the output from text into audio format. The listed URL's are being spoken by the system.

\subsection{Development}

The prototype was designed to operate on any internetconnected computer using Microsoft Windows $8 / 8.1$ as a single desktop application program after installation. The users of "An Audible Search Engine for Visually Impaired Users" have been given the instructions as follow:

- Audio input converted into text

- Audio media elements (shortcut keys were used)

- System search through net

- Tittles and links of search query were displayed

- $\quad$ Result selection

- $\quad$ Result open

In other words, An Audible Search Engine for Visually Impaired Users was designed as a World Wide Web browsing tool for efficient web links accessibility and usability for sightless or visually impaired individuals.

\subsubsection{Technical Tools}

- Coding: Microsoft Visual Studio 2013(Speech Recognition, Speech Synthesizer)

- $\quad$ Operating system: Windows 8.1

- Documentation: Microsoft Office 2013 
- Diagrams: Microsoft Visio 2013 Professional (Models)

\subsubsection{Prototype Interface}

\subsubsection{Instructions for Users}

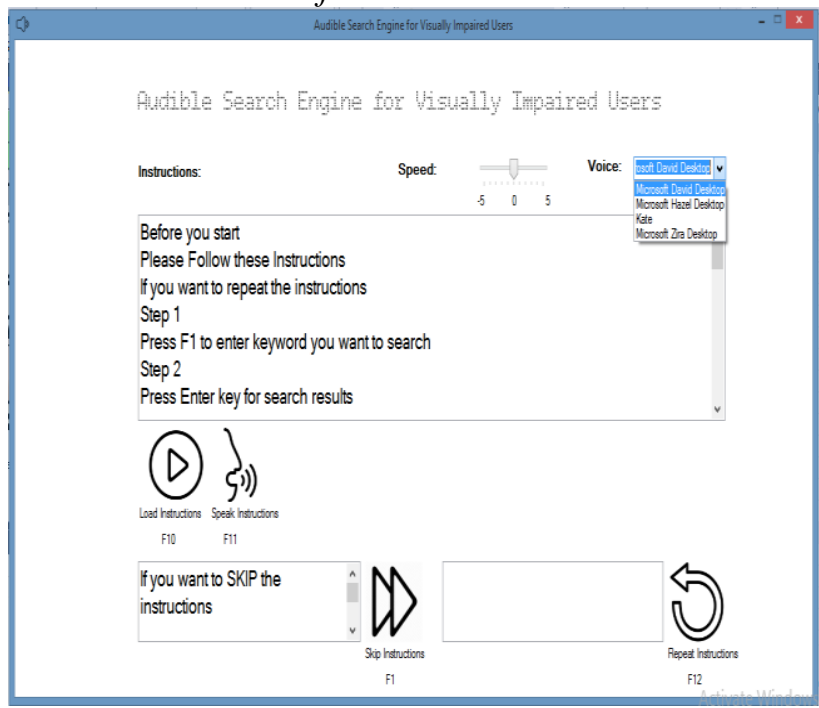

Figure 4. Search Engine Usage Instructions

Code for Short cut keys:

switch (keyData)

\{

case Keys.F1:

Skip();

return true;

case Keys.F10:

Start();

return true;

case Keys.F11:

Speak();

return true;

case Keys.F12:

Repeat();

return true;

\subsubsection{Search and Display Results Page}

Code for Search query:

private void SearchQuery()

string query $=$ tbQuery. Text;

var results $=$ Search(query);

listOfTitleAndLinks = new Dictionary<string,

string $>()$;

int count $=0$;

foreach (Result result in results)

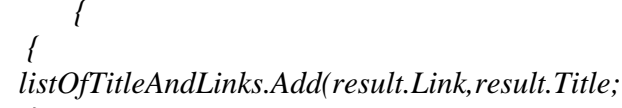

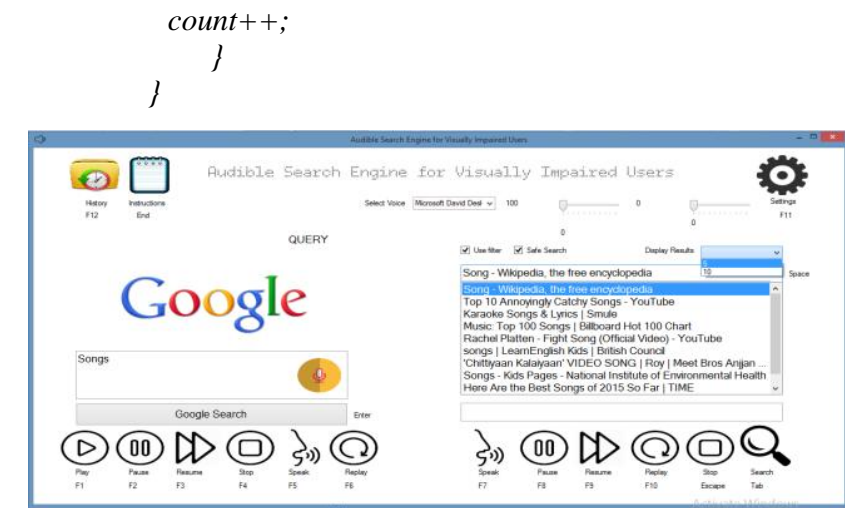

Figure 5. Search Engine Main Page with Available Facilities

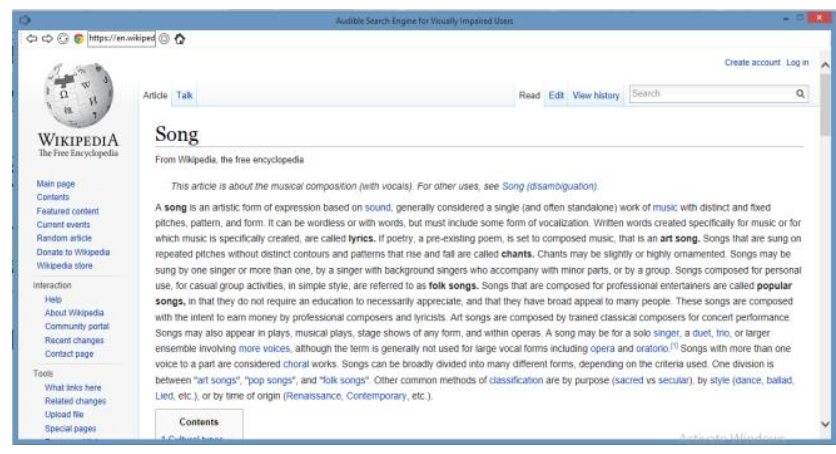

Figure 6. Search Results

3.6.2.3 Settings for Audio (Instructor of Visually Impaired User)

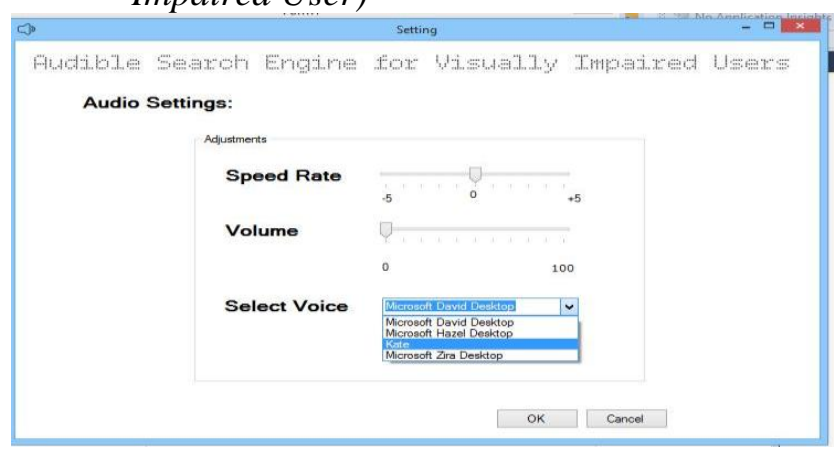

Figure 7. Interface for Audio settings

\section{DISCUSSION}

\subsection{How Other Search Engine Works}

4.1.1 Google Search Engine

Google released an accessible search for visually impaired users. In this technology, Google added an audio component in response to online petitions and outcry from visuallyimpaired users. It is modified for simple search and result exploration [7]. It works with the noise free environment but users have to click 'search by voice' which is difficult for the visually impaired users who have no experience of using Google before.

\subsubsection{Specialized Search Engine for the Blind} (SSEB)

A Specialized Search Engine for the Blind (SSEB) was developed with an accessible interface and improved functions i.e. searching assistance functions, user-centered functions, and specialized design for the blind. An experiment 
was conducted for both blind and sighted to verify the effects of SSEB. The performance was better with the SSEB than with the Google search engine, and the participants also showed higher satisfactions with the SSEB [14].

\subsection{How an Audible Search Engine for Visually Impaired Users works}

As compared to other search engines, an audible search engine for visually impaired users is an improved and modified version with simple search. This search engine application makes interaction easier and efficient by introducing shortcut keys for 'media elements events'. In addition, guidelines are provided in auditory form to sightless individuals before application usage. Moreover, users can also 'skip' or 'repeat' instructions according to their needs. It has simple and clear graphical user interface. Google search API and speech API's are used for users' voice recognition, search and results conversion into audio format. Speech recognition is used to know what user want to search and then it checked that word into the dictionary which is limited at the moment. After completion of word, user press 'search' shortcut key for searching that word. After searching through Google, it shows first 10 or 5 titles with relevant links by using Google search API. Speech synthesizer speaks out all the search results. User selects one of the title through given shortcut keys and web page relevant to selected title will open. Moreover, users have option of 'audio settings' and 'maintaining history'.

\section{LIMITATIONS}

An Audible Search Engine for Visually Impaired Users has capability to take audio input. It is also capable of writing texts through keyboard and users voice. Moreover, different media elements i.e. Play, Pause, Resume, Repeat, Speak, Stop are used to facilitate visually impaired individuals to interact with the system in an efficient way. In addition, guidelines are provided to the sightless users for significant help.

Moreover, some factors of this software are not improved i.e. low accuracy, and homonyms. Additionally, noisy environment is not feasible for this software. Moreover, vocabulary is limited for this designed software.

\section{CONCLUSION}

The research work presented in this paper demonstrates that usability for blind users can be greatly improved while maintaining an appealing search via web and speech recognition technology. To accomplish this, speech API's and Google search API were used by taking into account the problems of navigation via screen reader. First, it is described that how the blinds experience web search then analyzed a concrete example of search engine which offers audible input and output to assist visually impaired users.

The research work presented in this paper aims to explain the detailed analysis of search engine used by visually impaired users. It also presents the working of an audible search engine developed in C\#.Net. It discourses the role of all the components presented in finding information through web to alleviate visually impaired users.

\section{REFERENCES}

[1] A. Loo, M. Lu, C. Bloor, . "Internet Surfing for the Blind: A Prototype" in Journal of Electronic Library, Volume 21, 2003,pp. 575-586.

[2] M. Singh and P. Bhatia, "Automated Conversion of English and Hindi Text to Braille Representation" in International Journal of Computer Applications ,Volume

\section{4, July 2010, pp.0975 - 8887}

[3] Blenkhorn, paul [ble 1997], "A System for converting print into Braille ", IEEE Transaction on Rehabilitation Engineering,Vol. 3, No .2, June 1997, pp.215-221

[4] F. Reena Sharma and S. Geetanjali Wasson, " Speech Recognition and Synthesis Tool: Assistive Technology for Physically Disabled Persons" in International Journal of Computer Science and Telecommunications, Volume 3, Issue 4, April 2012, pp. 86-91.

[5] N. Ismail and H. Badioze Zaman, "Search Engine Module in Voice Recognition Browser to Facilitate the Visually Impaired in Virtual Learning " World Academy of Science, Engineering and Technology International Journal of Computer, Electrical, Automation, Control and Information Engineering Vol:4, No:11, 2010.

[6] P. Andronico, M. Buzzi, C. Castillo, B. Leporini, "Improving Search Engine Interfaces for Blind Users: a case study", Universal Access in the Information Society , June 2006, Volume 5, Issue 1, pp 23-40.

[7] B. Leporini, P. Andronico, M. Buzzi, "Designing search engine user interfaces for the visually impaired" in W4A '04 Proceedings of the 2004 international crossdisciplinary workshop on Web accessibility (W4A), ACM, New York, USA, 2004, pp. 57-66.

[8] F. Winberg, "Contextualizing Accessibility: Interaction for Blind Computer Users",Human - Computer Interaction, MDI, Stockholm: KTH, 2008. , pp. 88.

[9] Ummuhanysifa U, N. Banu, "A. Rahman, Voice Based Search Engine And Web Page Reader", International Journal of Computational Engineering Research, May 2013, pp. 1

[10] A. Al-Rehili, D. Al-Juhani, M. Al-Maimani and M. Ahmed, " A Novel approach to convert speech to Text and Vice-Versa and Translate from English to Arabic Language ", international Journal of Science and Applied Information Technology, Volume 1, No.2, May - June 2012.

[11] N.Karthik et al. " Voice Enabled Ontology Based Search Engine on Semantic Web For Blind", International Journal of Computer Science \& Engineering Technology, Vol. 5, No. 04, Apr 2014, pp. 341-344.

[12] Ferworn and H. Mark et al., "Auditory WWW Search Tools.", www.icad.org ICAD2000, Atlanta,USA, 2000 pp. 2-5.

[13] Y. Yang, S. Hwang,B. Schenkman,"An improved Web search engine for visually impaired users", Universal Access in the Information Society, 2012, pp. 113-124.

[14] Y. Yang et al." Specialized Design of Web Search Engine for the Blind People. ", Universal Access in Human-Computer Interaction. Applications and Services,Beijing, China,Springer Berlin Heidelberg, 2007.

[15] B. Singh, N. Kapur and P. Kaur," Speech Recognition with Hidden Markov Model: A Review", International Journal of Advanced Research in Computer Science and Software Engineering, March 2012, Vol 2. 